\title{
Program Verification by Symbolic Execution of Hyperfinite Ideal Machines (Extended Abstract)
}

\author{
James M. Morris and Mark Howard* \\ Odyssey Research Associates \\ 301A Harris B. Dates Drive \\ Ithaca, New York 14850-1313 \\ jmorris\%oravax.uucp@cu-arpa.cs.cornell.edu
}

\section{Introduction}

This paper describes Ariel, a program verification system based on the symbolic execution of hyperfinite ideal machines. By ideal machine we mean a transition system that gives an operational semantics for the language in which the programs we wish to verify are written. The terms of a transition system can be thought of as the states of an ideal (mathematical as opposed to electronic) machine, tailored to the language in question, which executes the program to be verified. The meaning of the program is identified with the execution traces of the transition system or ideal machine. A program is verified by showing that its traces satisfy its specification.

Our transition systems are defined in an applicative programming language for which we have a theorem prover. This applicative language, called Caliban, is lazy, higher-order, and polymorphic. The theorem prover, called Clio, provides a meta-language for making assertions about the meanings of Caliban terms. One can write a Caliban expression whose meaning is the trace of a (sub-)program beginning in some initial state. The preand postconditions that the initial and final states of such a trace respectively are to satisfy are written in Clio's meta-language. A (sub-)program is verified by using Clio to show that its precondition implies its postcondition. Clio's proof rule for implication attempts to prove the postcondition under assumption of the precondition. As Clio's prover is basically a term rewriting engine, proving the postcondition is basically a matter of expanding the symbols in the postcondition until no more expansion is possible. Here two properties of Caliban are important. First, Caliban is lazy. It is possible to write Caliban terms that represent non-terminating computations. Only those components of a term (state) of the transition system needed to determine that term's (state's) successor are actually evaluated. This is important for verifying sub-programs independently of their invocations. Secondly, Caliban expressions are executable. This means that much

"Funded by the U.S. Air Force, RADC contract F30602-86-C-0116 and the STARS program contract BOA \#3695.STARS-043. 
of the calculation involved in determining the next state of an Ariel transition system can actually be carried out automatically by Clio, which has the effect of reducing the amount of "bookkeeping" that must be dealt with in an Ariel verification. Ariel can execute programs symbolically. This provides us with the means to define a trace that represents all those traces in which the program's variables have values satisfying its precondition.

A hyperfinite ideal machine is a transition system in which some of the components of terms are defined using constructs from non-standard analysis. The original version of Ariel was designed primarily for the verification of numerical programs written in a subset of $\mathrm{C}$. We formulated a notion of correctness of numerical programs, called asymplotic correctness, using concepts from non-standard analysis (see [5] for a report on the theory of asymptotic correctness). This led to the formulation of non-standard models of computation which have proved to be of general utility in program verification.

The theory underlying Ariel uses non-standard analysis to formalize intuitive notions of what it means for a numerical program to compute a correct result. Numerical programs are not usually handled by verification systems because a sufficiently abstract yet tractable model of real arithmetic is difficult to design. When one attempts to model the amount of error introduced into a numerical calculation by finite arithmetic, one quickly gets bogged down in the intricacies of numerical analysis. The sort of verification we're interested in starts out with a program that purportedly implements some numerical method. We provide the means for showing that the program computes the desired result when run on a sufficiently accurate machine, i.e. that it's logically correct. Logical correctness turns out to be equivalent to asymptotic correctness. Asymptotic correctness captures programming errors that don't involve the magnitude of the error in computed results. Simple examples of such errors are : the sign of some variable is wrong, an expression uses the wrong operator, etc. A more subtle logical error is the following: comparing the signs of two quantities for equality by multiplying them and checking whether the product is positive. If the quantities are of different sign but small enough that the multiplication underflows to zero, the sign comparison will be incorrect.

\section{The Use of Non-standard Analysis in Ariel}

A program is asymptotically correct if, given any measure of the accuracy of the result to be computed, there exists a machine which will compute the result to the degree of accuracy given. Note first that this is weaker than the sort of properties the numerical analysts deal with. Numerical analysis tries to answer questions about how much accuracy in the machine operations and (most importantly) in the representation of the input data is required in order that the program compute a result that satisfies a given accuracy requirement. The problem with numerical analysis is its difficulty: questions of the sort just mentioned are hard to answer in general.

A program that is asymptotically correct will not contain errors of a logical nature. So, if a program has been specified to implement some numerical method, the theory of asymptotic correctness can be used to show that the program implements the method, as long as the specification doesn't require of the results some particular accuracy.

Why not just posit an ideal computing device that has the mathematical reals as a data type? The operations of such a machine would then deliver exact results when operating on exact data. The trouble is no worldly machine has such a data type. 
Moreover, most numerical methods only compute exact results in the limit. Therefore, their (straightforward) implementations would be non-terminating on such a machine.

What we would like to model is an ideal machine whose representational power and accuracy are limited, but without having to say to what degree. Intuitively, we would like to say things about the model like "its operations compute results which are close to ideal when their operands are not too big", which is the translation, in our general setting, of the idea that the operations of a worldly machine compute results of a certain accuracy when their operands are not so large as to cause overflow.

Analysis is the mathematics of approximation. It formalizes, in a precise way, our intuitive notions of closeness or approximation. Consider continuity. Intuitively, a real function $f$ is continuous if, whenever two values $x$ and $y$ in its domain are close (approximately equal), then $f(x)$ and $f(y)$ are close. Conventional analysis formalizes this intuitive notion by paraphrasing it in terms of the epsilon-delta argument we all learned in elementary calculus: $f$ is continuous at $x$ if, for all $\epsilon>0$, there is a $\delta>0$ such that $|f(y)-f(x)|<\epsilon$ for all $y$ for which $|y-x|<\delta$. Non-standard analysis gives a more direct formalization by idealizing "close" by the non-standard notion of infinitely close([3] is a general introduction to non-standard analysis). The non-standard formalization of continuity is: $f$ is continuous at $a$ if, whenever $x$ is infinitely close to $a, f(x)$ is infinitely close to $f(a)$. The direct way in which non-standard analysis expresses intuitive notions makes it easier to understand than conventional analysis and more easily implemented in theorem provers. Non-standard analysis can be expressed naturally in terms of operations rather than predicates and with fewer quantifiers, which makes it especially compatible with term-rewrite systems like Ariel's theorem prover.

Non-standard analysis is formed by adding the new undefined predicate standard to the usual language of mathematics. Other formal counterparts of informal concepts of non-standard analysis are defined from the predicate standard. A real number is infinite, corresponding to the informal notion "large", if its absolute value is larger than any standard real number; a real number is infinitesimal, i.e. "small", if its absolute value is smaller than any standard positive real number. Two real numbers are infinitely close, i.e. "close", if their difference is infinitesimal.

Concepts that can be defined without use of the predicate standard are said to be internal. These are the concepts of conventional mathematics. Those which cannot be defined without use of standard are called external, because they formalize notions which are outside conventional mathematics. The point of non-standard analysis is that it provides precise rules for manipulating the external notions. The rules of most interest to us here are:

1. there exists a real number which is non-standard,

2. the collection of standard real numbers satisfies the same internal properties as the collection of all real numbers.

Rule 2 is called the transfer principal; it is central to the theory underlying Ariel.

Numerical programs are useful in spite of the fact that they only compute approximations to ideal results, because the results they compute (one hopes) are close enough to ideal, i.e. they compute "good" approximations. For a given program, how good the approximation is depends on the accuracy of the machine on which its executed. If the program is correct, we should get increasingly accurate results, as we run it on increasingly accurate machines and, "in the limit", we should get exact results. This is what 
it means for a program to be asymptotically correct. Using the standard formalization of continuity as a guide, we might paraphrase asymptotic correctness in terms of an epsilon-delta argument: for any desired degree of accuracy $\epsilon$ of the result of running a program $P$, there exists a degree of accuracy $\delta$ of representational power and arithmetic operations such that, whenever $P$ is run on a machine $M$ of accuracy $\delta$, the results will be within $\epsilon$ of the ideal ones. Such a formalization would be as unwieldly to reason with as-it is to state. Instead, we take the non-standard formalization of continuity as our guide. We let the machine $M$ of the preceeding paraphrase be hyperfinite - the non-standard version of finite. In particular, its real data type is a hyperfinite subset of the non-standard reals. M's real data type will contain all the standard real numbers plus nonstandard reals infinitely close to each standard real. The non-standard values close to a standard real number $x$ correspond to sequences of reals that converge to $x$. In our theory such sequences are the approximations computed by a program when run on machines of increasing accuracy. We then restate asymptotic correctness: a program $P$ is asymptotically correct if it delivers results that are infinitely close to ideal when run a hyperfinite machine with finite data. This restatement is justified by the transfer principle. Let $\mathcal{M}=<M_{0}, M_{1}, M_{2}, \ldots>$ be a sequence of ideal machines of increasing accuracy and $\mathcal{R}=\left\langle r_{0}, r_{1}, r_{2}, \ldots\right\rangle$ be the results of running $\mathrm{P}$ on the members of $\mathcal{M}$. If executing $\mathrm{P}$ on a hyperfinite member $M_{\omega}$ of $\mathcal{M}$ produces a result $r_{\omega}$ which is infinitely close to ideal (let $r$ denote the ideal result), then for any standard real value $\epsilon$, the following property is true of $\mathcal{R}\left({ }^{*} \mathrm{~N}\right.$ is the non-standard natural numbers)

$$
\Phi\left(\epsilon,{ }^{*} \mathrm{~N}\right) \leftrightarrow \exists n \in{ }^{*} \mathrm{~N} \forall m \in{ }^{*} \mathbf{N}\left(m \geq n \rightarrow\left|r-r_{m}\right|<\epsilon\right)
$$

By transfer, $\Phi(\epsilon, N)$ is true. Since $\epsilon$ is arbitrary, $\Phi(\epsilon, N)$ is asymptotic correctness.

\section{Clio}

Ariel is built around a theorem proving system called Clio. Clio is designed for proving properties of expressions written in a lazy higher-order polymorphic applicative language called Caliban. Caliban is similar to Haskell and Miranda. Theorems about Caliban expressions are stated in Clio's metalanguage, which is essentially first-order logic with Caliban expressions as terms. Clio's prover is based on term rewriting augmented with other proof techniques such as structural induction, case splitting over variables of constructed type, fixed point induction, etc.

\subsection{Caliban}

Caliban is so similar to the functional languages mentioned above that we needn't describe much of it here. We mention in passing that a lazy applicative language like Caliban is an apt notation for specifying operational semantics. Caliban's constructed types and definitions by pattern matching provide the means for creating very concise descriptions of the components of ideal machines. In addition, the fact that Caliban type constructor functions are taken to be non-strict means that infinite objects, such as the trace of a non-terminating computation, can be defined.

Since it was designed to be a specification language, Caliban also provides a means for working with arbitrary sets. A set together with a bottom element becomes a flat 
domain called a sort. A sort is introduced by declaring its name along with the names and signatures of the functions on it. Properties of the sort and functions are specified by means of axioms. The functions are assumed to be strict and total. The non-standard real numbers we work with in Ariel are represented as a sort.

\subsection{Clio's Metalanguage}

Clio's metalanguage is essentially the language of first order predicate calculus with Caliban expressions as terms and bounded quantifiers that range over Caliban types. For Ariel's purposes, atomic formulas are of the form $A=B$, where $A$ and $B$ are Caliban expressions enclosed in back quotes. The usual logical connectives are available. Both universal and existential quantifiers are available. All quantifiers are bounded, i.e. they are of the form $(x:: \tau)$ or $(E x:: \tau)$ where $\tau$ is a Caliban type. Metalanguage predicates may be defined.

\subsection{Clio's Prover}

Clio's prover is basically a term rewriting engine. Whenever an assertion is proved or assumed it is made into a collection of rewrite rules which are added to Clio's rule base. Rules are used in two ways, as conditional rewrite rules and in a limited form of resolution (unit clause resolution when the result is a ground clause). Clio's rule manager also allows the user, interactively, to instantiate existing rules and to expand them (replace symbols by their definitions).

The basic strategy used by the prover is to skolemize an assertion to be proved and then reduce it to conjunctive normal form, i.e. a conjunction of clauses each of which is a disjunction. Each disjunct is a (possibly negated) atomic formula. The prover then attempts to prove each atomic formula by rewriting its left and right sides to the same normal form.

The prover's basic strategy is augmented by a number of additional proof techniques. If the assertion is a conjunction, Clio will break it up into its constituent conjuncts, each of which will have to be proved. If the assertion is an implication, Clio will assume the antecedent and add the corresponding rules to the rule base and then present the consequent to the user to prove (or try to prove it automatically). Existential assertions may be proved by supplying a witness to replace the quantified variable and then proving the resulting assertion. Proof by contradiction may also be used. Universal sentences may be proved either by generalization or structural induction (if the type of the variable of quantification is a constructed type). Disjunctions are proved by proving one of the equivalent assertions: $\neg B \Rightarrow A$ or $\neg A \Rightarrow B$, if the original assertion was $A \vee B$. Negations are proved by first pushing the negation inside the assertion. Then the usual suite of techniques is made available. A case split is available; one may case split both on Caliban and metalanguage expressions. 


\section{The Ariel Formalization of Numerical Computa- tion}

We formalize numerical computations as execution traces of an abstract interpreter. We do so for a number of reasons. First, we intend to expand our semantic model to include concurrent execution and the verification techniques for concurrent programs we find most attractive are based on operational models. Second, we wanted to model, to a certain extent, the possibility that a program was executed on a distributed system comprised of machines with different arithmetic, e.g. different rounding rules. On such a system the time at which a value was computed becomes significant - the approximate value resulting from evaluation of a given expression in the same context would, in principle, be different for different times. Two such values would differ only by an infinitesimal, but they wouldn't be the same. Third, an operational formalization seems most compatible with the concepts of non-standard analysis we use to model real arithmetic. The problem has to do with ensuring that one does not attempt to prove external properties of an execution trace by induction or by means of a proof rule whose soundness depends on an induction on the length of traces. An example of the latter is the theorem about while-loops that says that if $I$ is an invariant of the loop body when the loop guard is true, then $I$ and the negation of the loop guard is true when the loop terminates (if it does).

The semantic model used by Ariel is a transition system along the lines of G. Plotkin's work on structured operational semantics [4]. Ariel is based on an abstract interpreter, i.e. a transition system whose configurations consist of a syntactic representation of a program and some data that represent the current state of the program's execution. A program is represented by its abstract syntax tree coded as a Caliban constructed type. A program's static semantics are checked by the parsing program that builds its abstract syntax tree. The static semantic property most pertinent to execution is the resolution of ambiguous identifier references. In an abstract syntax tree an applied occurrence of a program variable is replaced by the abstract syntax tree for the declaration at the binding occurrence of the identifier determined by the scope rules of the language in which the program is expressed. Clio actually implements this replacement as a reference, so the size of the abstract syntax tree does not grow.

A state of the interpreter consists of a stack of procedure activation records called a context. Each activation record consists of a representation of which procedure corresponds to the activation record, where control is in that procedure, a partially evaluated expression, and a local store. As an expression is evaluated, its constituent parts are replaced by the results to which they evaluate. A store is a function from abstract locations to machine values (represented by its graph - a list of location-value pairs). A context is an element of a Caliban constructed type.

A next-state function is defined in Caliban. Traces of the abstract interpreter are defined by iterating the next-state function starting in some initial state. Thus, the meaning of a program or a piece of a program can be thought of as a function from initial states to traces. The next-state function may be iterated a fixed number of times or until some point in the program is reached depending on what one wants to verify. Since Caliban is a lazy language, just those parts of a trace required to prove a program's specification will actually be computed.

To illustrate how proofs of asymptotic correctness are mechanized, we must first de- 
scribe the formalizationm of real arithmetic. The natural numbers are a built-in Caliban type, MAT. They are modelled as a constructed type generated by the zero-ary constructor ZERO and the strict unary constructor SUCC:

$$
\text { NAT ::= Zero|Succ !NAT }
$$

(The ! indicates strictness in an argument.) Operations on elements of MAT are defined by recursion in the usual way. Caliban also contains built-in types of truth values, BooL, and characters, CHAR. Ariel uses the latter two Caliban types for its universes of truth values and characters, respectively. Ariel's integers are constructed from MAT. The Caliban definition is

$$
\text { INT ::= Intzero | Pos !NaT | Keg !NaT }
$$

Note that Pos Zero is the first positive integer, namely 1 , and analogously for Heg Zero. The successor and predecessor functions on IHT are defined in terms of the successor function on NAT in the obvious way.

The non-standard reals are formalized as a Caliban sort. Recall that a Caliban sort is essentially a set (made into a flat domain by appending $\perp$ below all the set's elements) plus functions on the set which are constrained to be strict and total. Sorts are incorporated into the Caliban domain by making their elements new atomic elements of the latter. The definition of the non-standard reals as a Caliban sort is as follows.

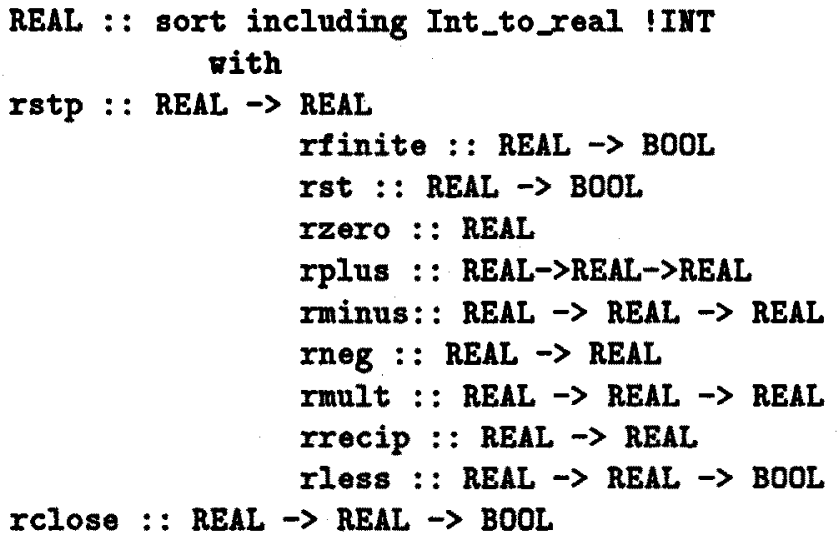

The including Int_to_real ! IrT clause indicates here that the sort contains all the integers plus additional elements; Int_to_real may be thought of as an injection function which also may be used as a constructor. It may be used in definitions by pattern matching, for example.

The functions on REAL are declared to satisfy a set of axioms - the usual axioms for the reals, minus the least-upper-bound axiom (which property the non-standard reals lack).

The axioms for non-standard analysis are based on the standard-part map, rstp, and the predicates rfinite, rst, and rclose (finite, standard and close, respectively). Ideally, there should be a single, polymorphic, undefined predicate standard, which should commute with applications of standard functions. This would yield a completely general transfer principle. However, such a predicate could not, in general, be continuous on non-flat domains such as function spaces, since its standardness would have to depend 
on only finitely many of its values, which is not the case. Therefore, it could not be part of the Caliban domain. Instead, our axioms model consequences of the transfer principle. One of these consequences is that the usual operations on the reals (plus, times, reciprocal, max, min, etc.) commute with the standard-part map. For example,

\section{AXIOY}

$(x)(y)^{\prime}$ rstp (rplus $\left.\times y\right)^{\prime}='$ rplus $($ rstp $x)($ rstp y)'

(The back quotes set off Caliban expressions in metalanguage formulae; $(x)$ and $(y)$ indicate that $x$ and $y$ are universally quantified.) The are similar axioms for the other arithmetic operations. Other axioms give the relationships between the standard-part map and the predicates mentioned above. For example,

\section{AXIOM}

$$
(x)(y)^{\prime} \operatorname{rclose} \times y^{\prime}={ }^{\prime}(\operatorname{rstp} x)=(\operatorname{rstp} y)^{\prime}
$$

As our main purpose here is to describe our program verification system, we will omit further details of our formalization of non-standard analysis.

We have specified that our hyperfinite ideal interpreter computes results of real arithmetic operations that differ from the ideal ones by an infinitesimal. This state of affairs is modelled by a function, machine, of type [NAT] $\rightarrow$ REAL $\rightarrow$ REAL. The first argument is to be interpreted as a time stamp, i.e. when the value was computed. Time stamps are represented as lists of natural numbers for the following reason. Each activation record in a context contains a count of the number of steps performed so far in the interpretation of the procedure corresponding to the activation record. The global time of the interpreter can conveniently be taken to be the ensemble of these local times collected together into a list in the order in which they appear in the context. Such a representation of global time turns out to be useful for differentiating the multiple incarnations of local variables that arise when a procedure is activated recursively. The second argument to machine is the ideal value approximated by the machine value. The meaning of this function is captured by the following axiom:

\section{AXIOM 'rstp (machine $n x)^{\prime}='(\operatorname{rstp} x)^{\prime}$.}

\section{Symbolic Execution Using Clio}

The basic difference between the Ariel approach to symbolic execution and others(cf. [2]) is that Ariel executes symbolically a complete semantic model of the program to be verified. Each state of an Ariel ideal machine represents all pertinent semantic information and each state of a symbolic execution trace is available directly to the theorem prover. The utility of having this much information available is that it allows us to verify any program written in the language supported, e.g programs that use aliasing or constructs for which proof rules or predicate transformers are not available. It allows us to verify security or real time properties which require reasoning about of sequences of states. It allows us to prove total correctness.

The Ariel system is interactive and integrated with its theorem prover, Clio. Given a specification for a procedure, one verifies it by proving that its symbolic execution trace satisfies its specification. If the program is specified by a precondition and a postcondition, one proves that the final state of such a trace satisfies the postcondition under the 
assumption that the initial state satisfied the precondition. This is presented to Clio as the assertion that the precondition implies the postcondition. Clio's built-in proof rule for implication causes the precondition to be assumed and a proof of the postcondition to be started; assumption of the precondition causes it to be transformed into a collection of rewrite rules.

The first step Clio takes to prove the postcondition is to send it to the reducer to be put into normal form. This invokes symbolic execution. A typical postcondition might say that the value returned by a function-procedure call (perhaps via a parameter) has some property. Assume, for the sake of discussion, that the postcondition of the procedure we wish to verify is

Post ' $x$ ' 's' ' $v$ ' := 'Returnstate $x s$ ' = ' $v$ '

where Return_state is a Caliban function that iterates (in the sense of repeated function application) the next-state function beginning in state $s$ until the current command is a return. Assume $x$ is one of the procedure's parameters and $v$ denotes some machine value. Clio puts Post into normal form by applying to it all the applicable rewrites in its rule base. When the definitions specifying the Ariel abstract machine are compiled by Clio, they are transformed into rewrite rules. So, the process of normalizing Post is essentially a matter of carrying out the repeated application of the next-state function beginning with a rule base that reflects the assumptions of the precondition. If the procedure to be verified consists of no more than a sequence of assignment statements, then when Post is normalized, the store of the resulting state will reflect the effect of the sequence of assignments and the postcondition is proved by "looking up" $x$ in the final store and seeing whether its value is $v$. Clio should be able to do this automatically.

The usual treatment of conditional statements in symbolic execution is to bifurcate the current path in the symbolic execution tree and attach the conditional statement's guard and its negation to the pathconditions of the respective newly created paths. In the Ariel system, this is handled by a case split. Clio detects the need for a case split automatically, when it encounters a Boolean subexpression whose value it is unable to determine in the process of normalizing an assertion. What happens is that the truth of the subexpression is assumed (and made into rules) and the proof of the assertion continued and similarly for the assumption that the subexpression is false. Ariel represents the path condition of other symbolic execution schemes by Clio's rule base, which makes all Clio's rule manipulation facilities available for arguments involving the path condition.

Loops are treated as follows. An assertion is attached to the beginning of the loop statement. This assertion should state

1. that each time control is at the beginning of the loop, the loop guard is true,

2. some invariant having to do with what the loop is to compute, and

3. that some function of the variables manipulated by the loop is strictly decreasing.

We then prove that this assertion cannot hold of any unbounded trace of a hyperfinite interpreter. The proof uses a theorem to the effect that in any unbounded trace of a hyperfinite machine, there are two states in which all identifiers are assigned the same values. This contradicts 3 . Thus we first prove that the loop terminates provided that the invariance of the loop assertion can be established. Clio makes this into a rule (or rules). The postcondition for the loop should consist of 2 and the negation of 1, i.e. 
when the loop ends (because its guard evaluates to false), it establishes what we wanted to compute. This is familiar. We prove the postcondition by contradiction. Having assumed its negation, we then prove the loop assertion by induction to get the required contradiction. If parts 2 and 3 of the loop assertion are actually invariant, then the only problem is with a state in which the loop guard is false. In this case, symbolic execution is invoked from the state in which control is at the beginning of the loop for the $i^{\text {th }}$ time, which allows Clio to prove the postcondition - a contradiction. If, in the state at the beginning of the $i^{\text {th }}$ iteration, the loop guard evaluates to true, the induction step can be proved directly.

Subroutines may be verified independently. A subroutine is specified by giving a preand postcondition for it. Using Clio, one proves that the subroutine terminates in a state satisfying its postcondition when started up in a state satisfying its precondition. Having successfully carried out the verification, one has Clio make the specification into rewrite rules. The latter will essentially be conditional rewrites which add the content of the postcondition to the rule base, whenever it's possible to prove the precondition.

\section{Conclusions}

We have described Ariel and the approach to program verification upon which it is based. The full paper includes a sample verification of a numerical program. We have built a prototype verification system and are in the process of trying it out on a suite of production numerical routines. These routines implement a continuous simulation of a rocket trajectory. They comprise about 1400 lines of $\mathrm{C}$ code (translated from the original FORTRAN) and calculate such things as a solution to the two-body problem, a RungeKutta integration, a Newton-Raphson iteration to solve a version of Kepler's equation, etc. Our prototype verifys programs written in a subset of $C$; we are extending the $C$ system to handle Unix system calls and are in the process of designing a new system for (a subset of) Ada.

\section{Acknowledgments}

The notion of asymptotic correctness and the use of nonstandard analysis to formalize it is due to Ian Sutherland based on a suggestion of Richard Mansfield. We would like to acknowledge the other members of the Ariel group, past and present, who contributed to the work reported here: Ian Sutherland, Doug Hoover, and Steve Brackin. We thank Garrel Pottinger and an anonymous referee for their comments on an earlier draft of this paper.

\section{References}

[1] Mark Bickford, Charlie Mills, and Edward A. Schneider. Clio: An Applicative Language-Based Verification System. Odyssey Research Associates Technical Report TR 15-7. April, 1989.

[2] S.L.Hantler and J.C.King. An introduction to proving the correctness of programs. ACM Computing Surveys, 8(3):331-353, September, 1976. 
[3] Albert E. Hurd and Peter A. Loeb, An Introduction to Nonstandard Real Analysis. Academic Press, 1985.

[4] Gordon D. Plotkin. A Structural Approach to Operational Semantics. DAIMI FN-19. Computer Science Department. Aarhus University, Denmark. September, 1981.

[5] Ian Sutherland. A Mathematical Theory of Asymptotic Computation. Rome Air Development Center Technical Report RADC-TR-87-261, Air Force Systems Command, Griffiss Air Force Base, NY. 1987. 